\title{
PRODUÇÃO DE MUDAS DE CAFÉ 'CONILON' PROPAGADAS VEGETATIVAMENTE EM DIFERENTES NÍVEIS DE SOMBREAMENTO
}

\author{
“CONILON” COFFEE SEEDLING PRODUCTION \\ AT DIFFERENT SHADING LEVELS
}

\author{
Heder Braun ${ }^{1}$; João Henrique Zonta²; Julião Soares de Souza Lima³ ${ }^{3}$ Edvaldo Fialho dos Reis ${ }^{3}$
}

\begin{abstract}
RESUMO
O café conilon (Coffea canephora) é uma cultura de grande importância na agricultura brasileira e ocupa posição de destaque entre os produtos de exportação. $\mathrm{O}$ cafeeiro pode ser conduzido em ambientes de baixa luminosidade, pois apresenta uma baixa irradiância de saturação. $\mathrm{O}$ trabalho teve como objetivo avaliar o crescimento de mudas de café conilon em diferentes níveis de sombreamento e a luz plena. O experimento foi realizado no CCA-UFES, Alegre/ES, no ano de 2005, num delineamento inteiramente casualizado com 3 repetições por tratamento, sendo as parcelas constituídas de 15 plantas, sendo 5 úteis. Os tratamentos constituíram de diferentes níveis de sombreamento $(30 \%, 50 \%, 75 \%)$ e luz plena. Foram avaliados os seguintes parâmetros: Altura das mudas, diâmetro do caule, massa fresca e seca da parte aérea, volume de raiz e área foliar. Dentre os parâmetros avaliados, apenas o diâmetro do caule não foi influenciado pelos diferentes níveis de sombreamento, sendo que, para os demais parâmetros avaliados, maiores valores foram encontrados com $75 \%$ de sombreamento. A partir dos resultados encontrados, conclui-se que a $75 \%$ de sombreamento as plantas do café conilon apresentam crescimento vegetativo maior em relação aos demais níveis.

Palavras-chave: Coffea canephora, crescimento, luz plena.
\end{abstract}

\begin{abstract}
The conilon coffee (Coffea canephora) is a greatly important crop in the Brazilian agriculture. It occupies distinguishable position among the export products, besides contributing for the job in field. The coffee shrub can be cropped under low brightness environments, since it shows low irradiance saturation. The objective of this study was to evaluate the growth of the conilon coffee seedlings at different shading levels and full light. The experiment was carried out under an entirely randomized design with three replicates, as the plots consisting of 15 plants (five useful ones), in CCA-UFES, Alegre county-ES, Brazil. The seedlings were grown in tubes with $34 \mathrm{~mm}$ diameter and $125 \mathrm{~mm}$ length, with $55 \mathrm{~cm}^{3}$ capacity. The treatments consisted of different shading levels $(30 \%, 50 \%$, 75\%) and total light. The following parameters were evaluated: seedling heights, stem diameter, and both fresh and dry matter of the aerial part, root volume and leaf area. Among the evaluated parameters, only the stem diameter showed no influence of the different shading levels, whereas higher values were found at $75 \%$ shading level. Based on the results, it is concluded that $75 \%$ shading level is the condition under which those coffee plants show a higher vegetative growth, relative to the other shading levels and the seedlings under total light, therefore providing the establishment of more vigorous seedlings.
\end{abstract}

Key words: Coffea canephora, growth, total light.

\section{INTRODUÇÃO}

O café conilon (Coffea canephora) é uma cultura de grande importância na agricultura brasileira e ocupa posição de destaque entre os produtos de exportação, além de contribuir para o emprego no campo (Carvalho et al., 1998).

O cafeeiro conilon é uma planta diplóide $(2 n=22$ cromossomos), auto-estéril e alógama por autoincompatibilidade do tipo gametofítica Conagin

1 Universidade Federal de Viçosa, Dep de Fitotecnia, Viçosa, MG, Campus Universitário, email: hederbraun@ hotmail.com

2 Universidade Federal de Viçosa, Dep de Engenharia Agrícola, Viçosa, MG, Campus Universitário, email:joaozonta@ hotmail.com

3 Departamento de Engenharia Rural, Centro de Ciências Agrárias da Universidade Federal do Espírito Santo, email: limajss@yahoo.com.br

3 Departamento de Engenharia Rural, Centro de Ciências Agrárias da Universidade Federal do Espírito Santo, email: edreis@cca.ufes.br

Fecha de Recepción: 14 Julio 2007

Fecha de Aceptación: 28 Agosto 2007 
\& Mendes (1961), conforme descrito por (Partelli et al. 2006). O café conilon é cultivado em baixas altitudes, até $400 \mathrm{~m}$, principalmente, no Espírito Santo, Rondônia e Bahia.

A produção de mudas sadias e vigorosas é o primeiro passo para a formação de uma lavoura cafeeira produtiva. Para o Coffea canephora a propagação por via assexuada tem sido praticada em grande escala, pois garante a transmissão das características desejáveis da planta mãe, eleva o nível de produtividade da lavoura, uniformiza as plantas e a maturação, possibilita escalonar a colheita, melhora o tamanho e a qualidade dos frutos, reduz a brotação de ramos ladrões, estimula a formação de ramos produtivos, proporciona maior resistência à doenças e ainda permite a produção de mudas durante todo ano (Paulino et al. 1985).

O enraizamento das estacas ocorre em função dos fatores ambientais, o estado fisiológico, a maturação, o tipo de propágulo e a época de coleta, que influenciam, sobretudo, na capacidade e na sua rapidez (Gomes, 1987). Em geral, estacas tomadas de plantas jovens enraízam com maior facilidade que estacas tomadas de ramos mais velhos (Hartmann $\&$ Kester, 1976).

Os viveiros utilizados para produção de mudas de café são construídos de varios materiais. Todos, porém seguem um padrão de construção como: as laterais construídas com um material que permiti passar 50\% de luminosidade (bambu, sombrite, etc.), evitando insolação direta nas mudas; a cobertura pode ser alta ou baixa, deve ser transversal ao caminhamento do sol, sendo construído de diversos materiais, tais como: bambu, ripado, sombrite, entre outros, sempre procurando manter o sombreamento em torno de $50 \%$, sendo estas recomendações utilizadas a muito tempo por viveiristas no Estado do Espírito Santo.

Apesar dessas recomendações, alguns produtores resolveram formar mudas a pleno sol, visando a mais rápida adaptação das mudas no campo e economia com material para construção do viveiro. Porém, há certa dificuldade na manutenção da umidade do substrato, sendo mais intensa a irrigação para esta condição de viveiro.

Modificações nos níveis de luminosidade que uma determinada espécie está adaptada, pode acarretar diferentes respostas em suas características fisiológicas, bioquímicas, anatômicas e de crescimento. Assim, a eficiência do crescimento pode estar relacionada à habilidade de adaptação das plantas às condições de intensidade luminosa do ambiente (Engel, 1989; Kozlowski et al., 1991; Atroch et al., 2001).

Sabe-se que o cafeeiro é uma planta C3, ou seja, é uma planta de ambiente sombreado, que possui adaptações fisiológicas e morfológicas para isso, como a baixa irradiância de saturação, variando de 300 a $600 \mu$ mol fótons $\mathrm{m}^{-2} \mathrm{~s}^{-1}$ (Kumar \& Tieszen, 1980; Fahl \& Carelli, 1994). A adaptação das plantas ao ambiente de luz depende do ajuste de seu aparelho fotossintético, de modo que a luminosidade ambiental seja utilizada de maneira mais eficiente possível. As respostas dessa adaptação serão refletidas no crescimento global da planta. Assim, a eficiência do crescimento pode estar relacionada com a habilidade de adaptação das plântulas e as condições de intensidade luminosa do ambiente, freqüentemente as análises do crescimento são utilizadas para predizer o grau de tolerância das diferentes espécies ao sombreamento (Engel, 1989).

Várias características constituem parâmetros para avaliar as respostas de crescimento de plantas à intensidade luminosa. Dentre essas, a de uso mais freqüente é a altura das plantas, visto que a capacidade em crescer rapidamente, quando sombreadas, é um mecanismo de adaptação das plantas (Moraes Neto et al., 2000). Outros parâmetros bastante utilizados são o diâmetro do colo e a produção de massa seca. $\mathrm{O}$ crescimento em diâmetro depende da atividade cambial que, por sua vez, é estimulada por carboidratos produzidos pela fotossíntese e hormônios translocados das regiões apicais. Logo, o diâmetro de colo é um bom indicador da assimilação líquida, já que depende mais diretamente da fotossíntese (Engel, 1989).

Como exemplo, mudas de cacau obtiveram diâmetros do colo maiores em intensidades de $50 \%$ e $70 \%$ de sombra. A produção de massa seca permite avaliar o crescimento de uma planta, pois é reflexo direto da produção fotossintética líquida, somada à quantidade de nutrientes minerais absorvidos, $\mathrm{o}$ que corresponde apenas a uma pequena parcela daquela (Engel, 1989).

Atualmente, a maioria dos plantios comerciais de café conilon é realizada com mudas provenientes de estacas. Contudo, faltam informações conclusivas quanto ao comportamento comparativo de plantas propagadas por sementes e por estacas e, para estas últimas, há importantes questionamentos quanto ao desenvolvimento da planta, principalmente do sistema radicular, sua capacidade de crescimento 
em profundidade e sustentação de toda a planta (Partelli et al. 2006).

Como são escassos experimentos na formação de mudas de café a pleno sol, este trabalho teve como objetivo avaliar o desenvolvimento de mudas do cafeeiro Coffea canephora propagadas por estacas a pleno sol e sob diferentes níveis de sombreamento.

\section{MATERIAL E MÉTODOS}

$\mathrm{O}$ experimento foi conduzido em viveiro de produção de mudas do CCA / UFES, no município de Alegre, localizado na região sul do Estado do Espírito Santo. O local do experimento está situado a uma altitude média de 150 metros, latitude de $20^{\circ} 45^{\prime} 40,42^{\prime}$ ' S e longitude $41^{\circ} 32^{\prime} 06,73$ ' $\mathrm{W}$. A temperatura média da região é de $24,1^{\circ} \mathrm{C}$, com temperaturas média máxima e mínima de $31,0^{\circ} \mathrm{C}$ e $20,2^{\circ} \mathrm{C}$, respectivamente. $\mathrm{O}$ total precipitado no ano foi de $1429,4 \mathrm{~mm}$.

$\mathrm{O}$ viveiro utilizado possui cobertura alta e foi dividido em partes, nas quais foram colocados diferentes tipos de sombrite, compondo os tratamentos: área sem sombrite (Pleno Sol), sombrite de $30 \%, 50 \%$ e de $75 \%$ de sombra. Os canteiros foram dispostos no centro de cada área, cobertos de modo a não serem influenciados por outros tipos de sombreamento em nenhuma hora do dia.

As estacas para formação das mudas foram obtidas no município de Alegre - ES, de tecido adulto de ramos ortotrópicos de ponteiras do cafeeiro, sendo retiradas de plantações com bom aspecto fitosanitário e nutricional. Anteriormente ao plantio nos tubetes, as estacas foram enraizadas em caixa de areia. Após o enraizamento realizou-se o transplantio para os tubetes, de $34 \mathrm{~mm}$ diâmetro e $125 \mathrm{~mm}$ de comprimento, com capacidade de 55 $\mathrm{cm}^{3}$, contendo substrato comercial (Plantmax), os quais foram alocados em bancada suspensa.

$\mathrm{O}$ delineamento experimental utilizado foi o inteiramente casualizado, com 3 repetições por tratamento, sendo as parcelas constituídas de 15 plantas, sendo 5 úteis.

As irrigações foram feitas diariamente durante o período de 25 dias de enraizamento, e na fase de desenvolvimento vegetativo das estacas, durante um período de 140 dias, de forma manual, mantendo sempre a capacidade de campo do substrato para todos os tratamentos.
Foi realizado o controle nutricional e fitossanitário das mudas quando necessárias, de acordo com as recomendações propostas para esta cultura.

Para avaliar o desenvolvimento das plantas, as variáveis adotadas foram: altura das mudas, medida do colo das plantas até a gema apical; diâmetro do caule, medida no colo da planta com um paquímetro; massa fresca da parte aérea, após a lavagem para retirada de solo, secionou-se o caule na altura do colo; massa seca da parte aérea foi acondicionada em sacos de papel devidamente etiquetados com os números dos tratamentos e secas em estufa com circulação forçada, a $60^{\circ} \mathrm{C}$, até atingir peso constante. Pesou-se o material em balança de precisão. O volume de raiz, após lavagem, foi medido em proveta graduada. Para a determinação da área foliar utilizou-se o medidor LI - COR 3100.

Os dados obtidos foram submetidos à análise de variância e as médias foram comparadas pelo teste de Tukey a 5\% de probabilidade.

\section{RESULTADOS E DISCUSSÃO}

Dentre as características avaliadas, apenas o diâmetro do caule não sofreu efeito do sombreamento. Desenvolvimento significativo foi encontrado com $75 \%$ de sombreamento, para as demais características, demonstrando comportamento característico para o café conilon de plantas de ambiente sombreado.

De acordo com os resultados apresentados na Figura 1, a altura das mudas foi influenciada pelos níveis de sombreamento, sendo o tratamento com $75 \%$ o que apresentou maior valor, devido ao baixo índice de luminosidade, que acarreta mudanças morfofisiológicas comuns nas plantas cultivadas sob estas condições. Segundo Varela \& Santos (1992), citados por Almeida et al. (2005), trabalhando com Dinizia excelsa Ducke (angelim pedra) encontraram maiores valores em altura, diâmetro de coleto, e massa seca da parte aérea e radicular em mudas submetidas a sombreamento de 30 e $50 \%$ do que em $70 \%$. Portanto, os resultados obtidos no presente trabalho não estão de acordo com os encontrados pelos referidos autores.

De acordo com os dados referentes ao diâmetro do caule das mudas (Figura 2), estes não diferiram estatisticamente entre si. Mudas com alto valor de diâmetro de coleto indica que haverá boa taxa de sobrevivência após o plantio, conforme Almeida et al. (2005), uma vez que esse é um indicador 


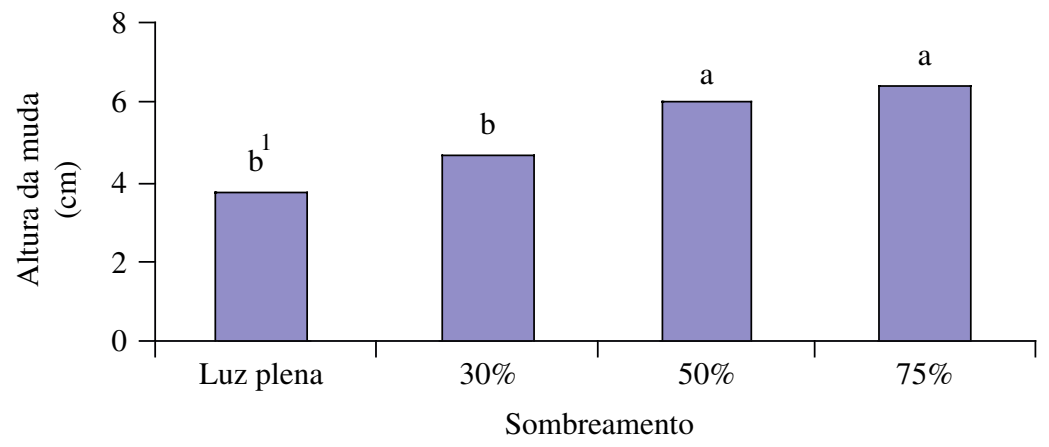

1 Médias seguidas pela mesma letra não diferem estatisticamente pelo teste de Tukey a 5\% de probabilidade. Figura 1. Altura das mudas de café conilon produzidas sob diferentes níveis de sombreamento.

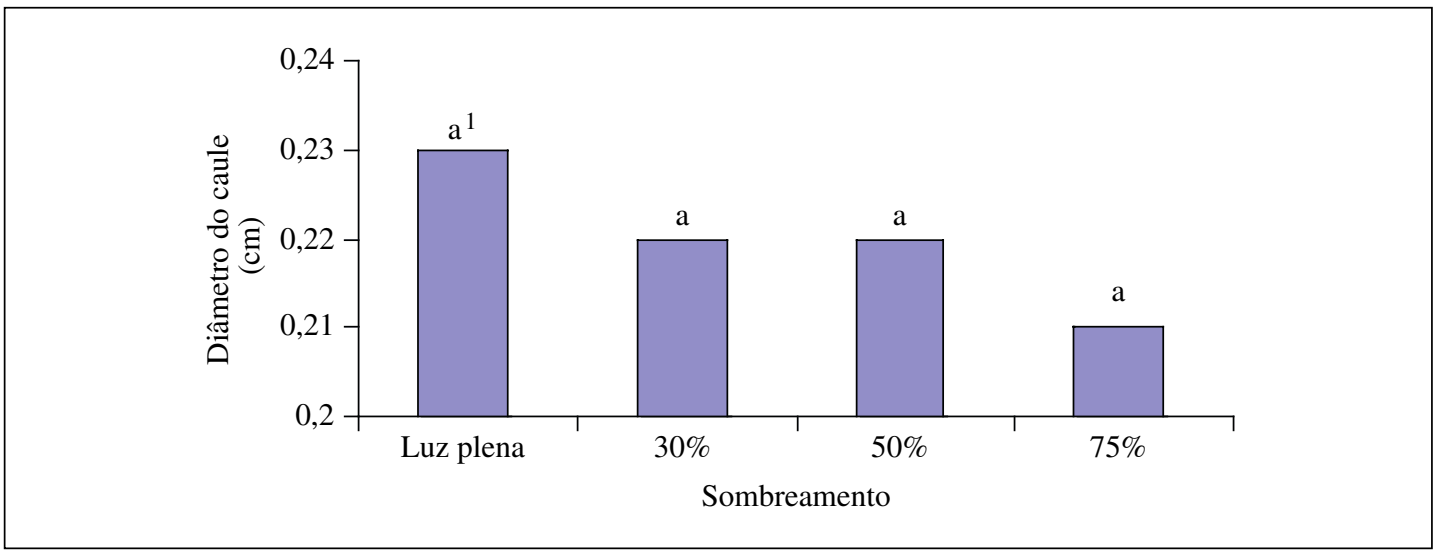

1 Médias seguidas pela mesma letra não diferem estatisticamente pelo teste de Tukey a 5\% de probabilidade.

Figura 2. Diâmetro do colo das mudas de café conilon produzidas sob diferentes níveis de sombreamento.

das taxas de assimilação liquida de produtos da fotossíntese (Gonçalves et al. 2000). Ricci et al. (2006) trabalhando com cultivo de café orgânico a pleno sol e sombreado, relatou que o sombreamento reduziu o diâmetro do caule dos cafezais.

Analisando os resultados referentes à massa fresca e seca da parte aérea das mudas do cafeeiro (Figura 3 e 4), os resultados diferiram estatisticamente entre si, sendo que as mudas provenientes do tratamento com luz plena não obtiveram um bom desenvolvimento. Portanto, nestas condições, a parte aérea não encontrou condições de se tornar uma muda que suporte todas as condições ambientais de campo. O tratamento com $75 \%$ de sombreamento foi o que apresentou os maiores valores de massa fresca e seca da parte aérea, apresentando mudas mais vigorosas, confirmando os resultados obtidos por (Kumar \& Tieszen, 1980), onde afirmam que o cafeeiro se desenvolve bem em ambiente de baixa luminosidade. Matiello et al. (1989), trabalhando com cinco níveis de sombra, variando de 0 a $100 \%$, em áreas com período seco acentuado no Nordeste brasileiro (Brejão-PE), verificaram que o sombreamento tem resultado em melhor enfolhamento e maior produtividade dos cafeeiros, sendo os melhores resultados obtidos com os níveis de 50 a $75 \%$ de sombra.

Os resultados referentes à massa seca da parte aérea estão de acordo com Paiva et al. (2003), que trabalharam com a influencia de diferentes níveis de sombreamento sobre o crescimento de mudas de cafeeiro (Coffea arabica L.), obtendo-se maiores valores com o nível de $90 \%$ de sombreamento. Carelli et al.(2001) trabalhando com Coffea arabica 


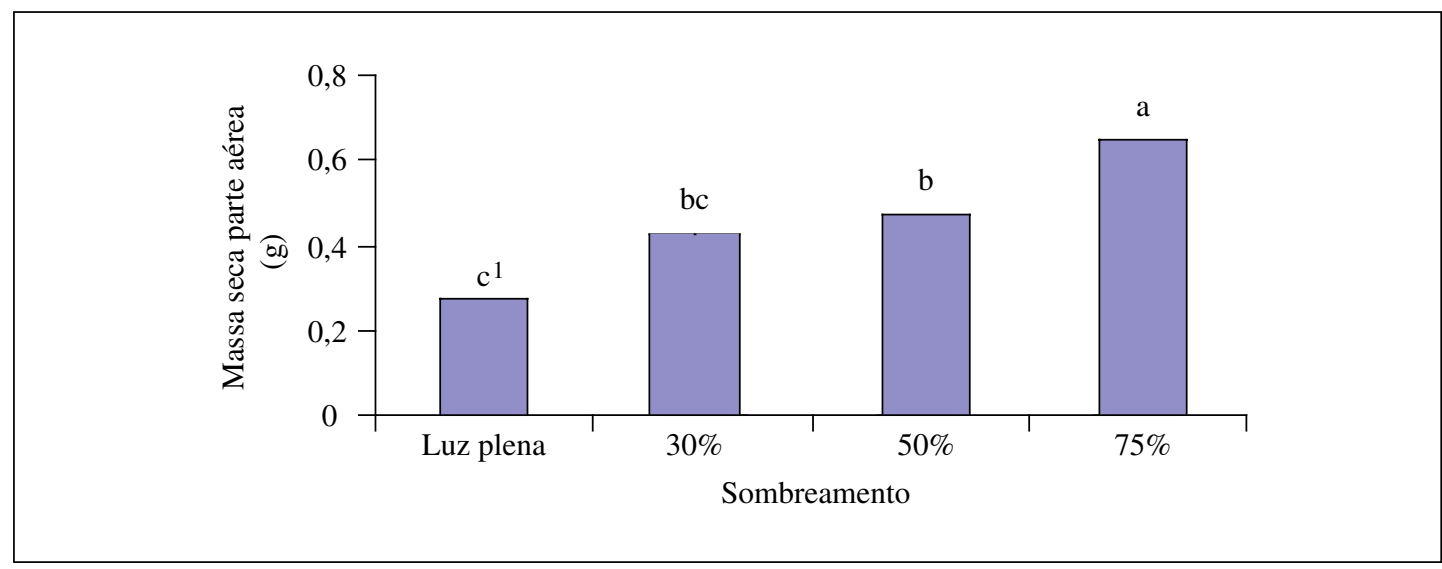

1 Médias seguidas pela mesma letra não diferem estatisticamente pelo teste de Tukey a 5\% de probabilidade.

Figura 3. Massa seca da parte aérea de mudas de café conilon produzidas sob diferentes níveis de sombreamento.

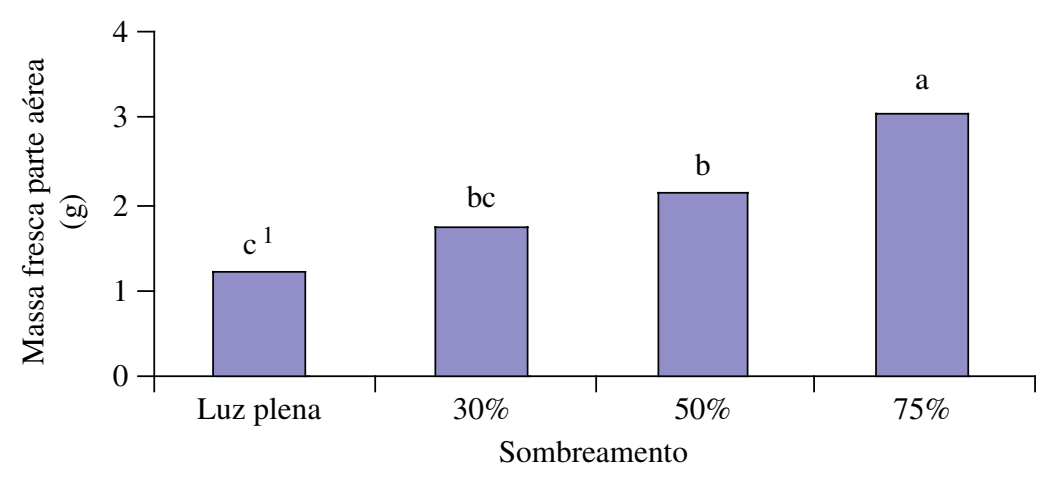

1 Médias seguidas pela mesma letra não diferem estatisticamente pelo teste de Tukey a 5\% de probabilidade.

Figura 4. Massa fresca da parte aérea de mudas de café conilon produzidas sob diferentes níveis de sombreamento.

cv. Obatã verificaram que a massa seca aumenta em sombreamento com relação à plena luz.

Observando os resultados referentes à área foliar (Figura 5), nota-se que as mudas produzidas sob $75 \%$ de sombreamento obtiveram maiores valores, apresentando diferença significativa entre os níveis de sombreamento $(50$ e $75 \%)$ e á plena luz. Esses resultados também foram encontrados por Ricci et al. (2006), observando que pode ser um mecanismo utilizado pelo cafeeiro para compensar a menor luminosidade recebida. Resultados obtidos em diversos cultivares mostraram que o cafeeiro, quando cultivado em condições sombreadas, desenvolve folhas mais finas e maior área foliar, que proporcionam maior interceptação da luz disponível (Fahl et al., 1994).
Nos resultados referentes ao volume de raiz (Figura 6), pode-se observar que houve diferenças estatísticas entre os níveis de sombreamento e o à plena luz, sendo que o tratamento com $75 \%$ de sombreamento apresentou maior volume de raiz, e as mudas que estavam em pleno sol apresentaram menor volume. Esses resultados provavelmente se devem ao fato de as mudas produzidas sob $75 \%$ de sombreamento apresentarem maior área foliar, vindo de encontro aos resultados obtidos por Biase \& Costa (2003), que trabalhando com propagação vegetativa de Lippia alba (erva cidreira do campo) com diferentes estacas, relataram que houve diferenças estatísticas entre os tipos de estacas estudadas, sendo que as estacas com maior numero de folhas apresentaram maior volume de raiz. 


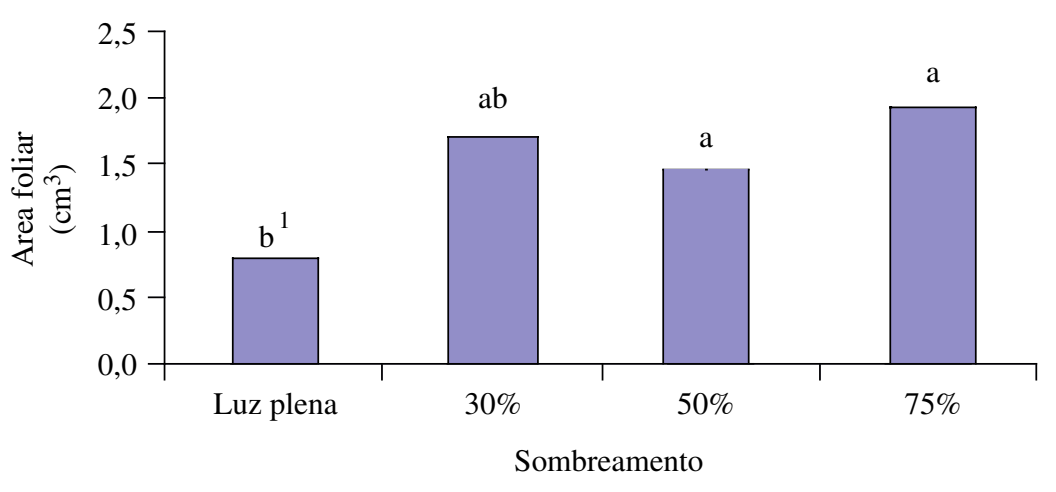

1 Médias seguidas pela mesma letra não diferem estatisticamente pelo teste de Tukey a 5\% de probabilidade. Figura 5. Área foliar das mudas de café conilon produzidas sob diferentes níveis de sombreamento.

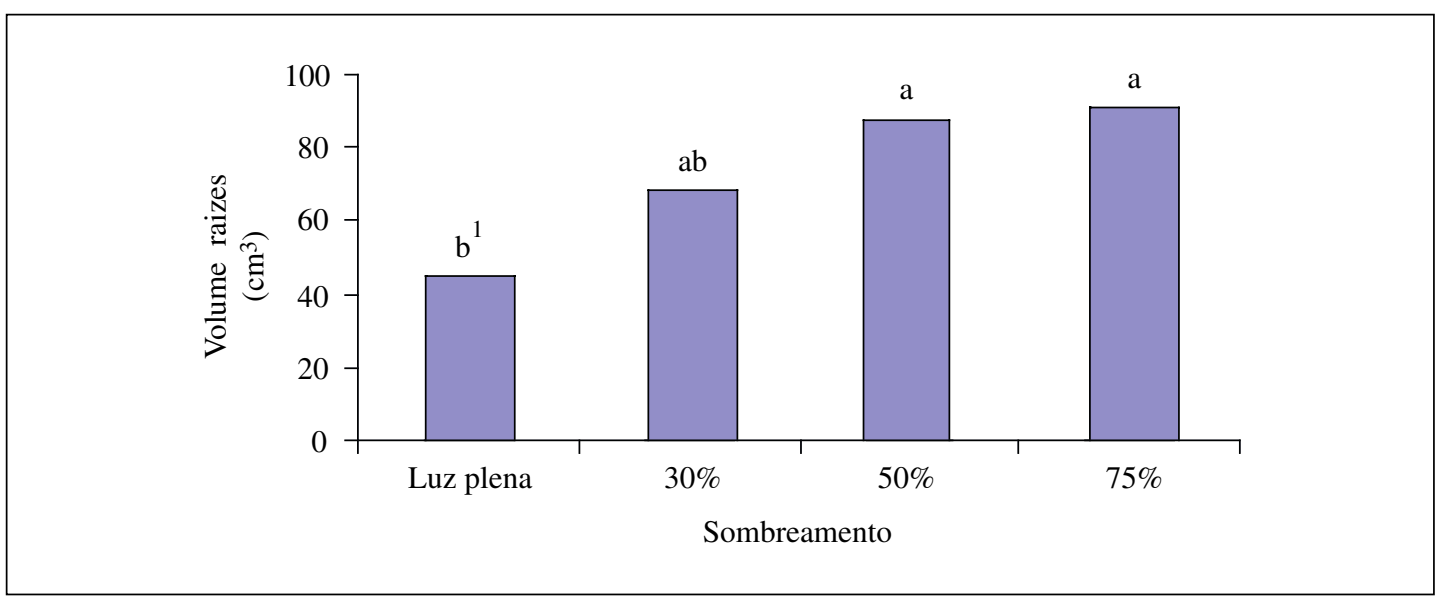

1 Médias seguidas pela mesma letra não diferem estatisticamente pelo teste de Tukey a 5\% de probabilidade. Figura 6. Volume de raiz das mudas de café conilon produzidas sob diferentes níveis de sombreamento.

Os níveis mais elevados de sombreamento (75\%) propiciam uma melhor condição climática às mudas, protegendo-as do estresse provocado por elevadas temperaturas e perda de umidade do substrato, reduzindo a evapotranspiração (Boulay et al., 2000), evitando, desta forma, um maior desfolhamento durante a estação seca, aumentando assim a massa seca da parte aérea.

\section{LITERATURA CITADA}

ALMEIDA, L.S. DE; MAIA, N.; ORTEGA, A.R.; ÂNGELO,

A.C. 2005. Crescimento de mudas de jacarandá puberula cham. em viveiro, submetidas a diferentes níveis de luminosidade. Ciência Florestal, v. 15 n. 3, p 323-329.

\section{CONCLUSÕES}

$75 \%$ de sombreamento é a condição na qual as mudas do café conilon apresentam maior crescimento vegetativo em relação aos demais níveis de sombreamentos, propiciando a formação de mudas mais vigorosas, com maiores condições de se adaptar em campo no momento do transplantio.
ATROCH, E. M. A. C.; SOARES, A,M.; ALVARENGA, A.A. DE; CASTRO, E.M. DE. 2001. Crescimento, teor de clorofilas, distribuição de biomassa e características anatômicas de planas jovens de Bauhinia forficata Link 
submetidas a diferentes condições de sombreamento. Ciência Agrotecnologia, v. 25, n. 4, p. 853-862.

BIASE, L. A.; COSTA, G. 2003. Propagação vegetativa de Lippia alba. Ciência Rural, v. 33, n. 3.

BOULAY, M.; SOMARRIBA, E.; OLIVIER, A. 2000. Calidad de Coffea arabica bajo sombra de Erythrina poeppigiana a diferentes elevaciones en Costa Rica. Agroforestería en las Américas, v. 7, p. 40-42.

CARELLI, M.L.C.; FAHL, J.I. SET. DE 2001. Efeitos do sombreamento em produtividade e crescimento. In: II Simpósio de Pesquisa dos Cafés do Brasil.

CARVALHO, E. A. M. DE; POZZA, E. A.; JULIATTI, F. C.; MOREIRA, J. C.; PEIXOTO, A. S.; SANTOS, C. M. DOS. 1998. Impacto dos diferentes sistemas de irrigação e lâminas d'água na evolução da ferrugem do cafeeiro. In: Simpósio Brasileiro de pesquisa em Cafeicultura Irrigada, 1. Araguari-MG. Palestras e resumos. Araguari: UFU, p. 101-103.

CONAGIN, C. H. T. M.; MENDES, A. J. T. 1961. Pesquisas citológicas e genéticas em três espécies de Coffear, autoincompatibilidade em Coffea canephora. Bragantia, v.20, p. 787-804.

ENGEL, V. L. 1989. Influência do sombreamento sobre o crescimento de mudas de essências nativas, concentração de clorofila nas folhas e aspectos de anatomia. Escola Superior de Agricultura "Luiz de Queiróz", Piracicaba, 202 p. (Dissertação de Mestrado).

FAHL, J.I., CARELLI, M.L.C. 1994. Influência do sombreamento nas características fisiológicas envolvidas no crescimento de espécies de coffea. In: Simpósio Internacional sobre Café Adensado, Londrina, Anais... Londrina: IAP, 1994. P. 289-290.

GOMES, A.L. 1987. Propagação clonal: Princípios e particularidades. Vila Real: Universidade de Trás-os-Montes e Alto Douro, 69 p. (Série Didáctica, Ciências Aplicadas, 1).

GONÇALVES, J.L.; SANTARELLI, E.G.; MORAES NETO, S.P. 2000. Produção de mudas de espécies nativas: substrato, nutrição, sombreamento e fertilização. In: Gonçalves \& Benedetti. Nutrição e fertilização florestal. Piracicaba: IPEF, p. 310-350.
HARTMANN, H.T.; KESTER, D.E. 1976. Propagacion de plantas, princípios e práticas. 5. Ed. México: Cia. Editorial Continental, $810 \mathrm{p}$.

KOZLOWSKI, T.; KRAMER, P.J.; PALLARDY, S.G. 1991. The physiological ecology of woody plants. London: Academic Press, $657 \mathrm{p}$.

KUMAR, D., TIESZEN, L.L. Photosynthesis in Coffea arabica Effects of light and temperature. Experimental Agriculture, Cambridge, v. 16, n. 1, p. 13-19, Jan. 1980.

MATIELLO, J.B.; DANTAS, F.A.S.; CAMARGO, A.P. DE; RIBEIRO, R.N.C. 1989. Níveis de sombreamento em cafezal na região serrana de Pernambuco: parte III. In: CONGRESSO BRASILEIRO DE PESQUISAS CAFEEIRAS, 15, 1989, Maringá, PR. Anais . Rio de Janeiro: IBC, p. 182.

MORAES NETO, S.P.; GONÇALVES, J.L. DE M.; TAKAKI, M.; CENCI, S.; GONCALVES, J. C. 2000. Crescimento de mudas de algumas espécies arbóreas que ocorrem na Mata Atlântica em função do nível de luminosidade. REVISTA ÁRVORE, VIÇOSA - MG, v. 24, n. 1, p. 35-46.

PAIVA, L.C.; GUIMARÃES, R.J.; Souza, C.A.S. 2003. Influência de diferentes níveis de sombreamento sobre o crescimento de mudas de cafeeiro (Coffea arábica 1.). Ciência e Agrotecnologia., Lavras. V. 27, n. 1, p. 134-140, jan./fev.

PARTELLI, F.L.; VIEIRA, H.D.; SANTIAGO, A.R.; BARROSO, D.G. 2006. Produção e desenvolvimento radicular de plantas de café 'Conilon' propagadas por sementes e por estacas. Pesquisa Agropecuária Brasileira., Brasília, v. 41, n. 6, p. 949-954, jun.

PAUlinO, A.J.; MATIELlO, J.B.; PAULINI, A.E. 1985. Produção de mudas de café conilon por estacas. Rio de Janeiro, RJ: MIC/IBC/GERCA, 12 p. (IBC/GERCA. Instruções técnicas sobre a cultura de café no Brasil, 18).

RICCI, M. DOS S. F.; COSTA, J.R.; PINTO, A.N.; SANTOS, V.L. DA S. 2006. Cultivo orgânico de cultivares de café a pleno sol e sombreado. Pesquisa Agropecuária Brasileira, Brasília, v. 41, n. 4, p. 569-575, abr.

VARELA, V. P.; SANTOS, J. 1992. Influência do sombreamento na produçao de mudas de Angelim pedra (Dinizia excelsa Ducke). Acta Amazonica, v.22, n. 3, p. 407-411. 